Auch im Pflegebereich geht nichts mehr ohne "Kollege“ Computer. Wir stellen Ihnen hier nicht nur die wichtigsten Software-Anwendungen für die Pflege vor, sondern beantworten auch Fragen

\title{
Dienstplanprogramme
}

\section{Personal im Einsatz}

\author{
Dienstpläne sollen verlässlich sein und den unterschiedlichen \\ Bedürfnissen der Mitarbeiter gerecht werden - keine leichte Aufgabe. \\ In den meisten Einrichtungen haben sich daher Softwarelösungen \\ durchgesetzt, die die Dienstplanung erleichtern.
}

$\mathrm{D}$ ienstplanprogramme bestehen in der Regel aus einer Software zur Personalverwaltung und einer zur Dienstplanung. In der Personalverwaltung werden für alle Mitarbeiter Stamm- und Vertragsdaten sowie tarifliche und gesetzliche Informationen hinterlegt. Damit kann das System den relevanten Planungsrahmen errechnen.

\section{Dienstplanung leicht gemacht}

Die Planung erfolgt auf einer kalenderähnlichen Oberfläche, in der Wochenenden und Feiertage bereits gekennzeichnet sind. Auch die für diesen Zeitraum zugeordneten Mitarbeiter werden zeilenweise angezeigt. $\mathrm{Zu}$ jedem Mitarbeiter kann der Dienstplaner Informationen einblenden und hat so Soll-Stunden und geplante Abwesenheiten im Blick. Plausibilitätskontrollen prüfen in Echtzeit die Einhaltung von Dienstvereinbarungen und Gesetzen, damit gesetzliche Ruhezeiten nicht unterschritten werden.

Auch die Zahl der Mitarbeiter pro Tag und Schicht wird automatisch errechnet und angezeigt. So lassen sich Planungslücken rasch erkennen und organisieren. Bei abgeschlosser Planung wird der Planungsstatus (Soll) versiegelt und der Ist-
Plan für die real geleisteten Dienste geöffnet. Abweichungen sind die Grundlage für die Berechnung der Minus- und Plusstunden.

Die Berechnung und tagesaktuelle Dokumentation von geänderten Diensten, Abwesenheiten und Abweichungen erlaubt es allen Mitarbeitern, ihre Überstundenstände oder Resturlaubstage jederzeit abzurufen. Oft wird den Mitarbeitern sogar ein eigenes Portal geboten, in dem sie zum Beispiel ihre Wünsche nach Mehr- oder Minderarbeit oder Urlaub eintragen und Auswertungen generieren können.

Bei der Kompensation von Ausfällen kann sich das Pflegemanagement am PC schnell einen Überblick über die Besetzungssituation aller Stationen verschaffen. Vielfältige Auswertungsmöglichkeiten, wie Urlaubsverteilung oder Ausfallstatistik, unterstützen das Pflegecontrolling. Die geprüften Dienstpläne der Stationen gehen zudem automatisch an die Lohnbuchhaltung. Dadurch werden Bearbeitungszeiten verkürzt und Dienstplanänderungen (z.B. Überstunden) können in der Lohnzahlung schneller berücksichtigt werden. Auch nachträgliche Korrekturen werden deutlich einfacher. Ein

\section{FAQ - HÄUFIG GESTELLTE FRAGEN}

Wie sind Dienstplanprogramme aufgebaut?

Diese Programme bestehen aus einem Dienstplanprogramm und einer Personalverwaltung mit allen relevanten Mitarbeiterdaten.

\section{Welche Vorteile bringen sie für das Pflegemanagement?}

Durch die automatisch Berechnung der Mitarbeiter pro Tag und Schicht lassen sich Planungslücken rasch erkennen und organisieren. Das Pflegemanagement hat immer einen Überblick über die Besetzungssituation sämtlicher Stationen. zu Datensicherheit und Datenschutz.

1.Pflegeinformationssysteme

2. Dienstplanprogramme

3. Patientendatenmanagement

4. Material- und Apothekenbestellung

5. Datensicherheit und Datenschutz

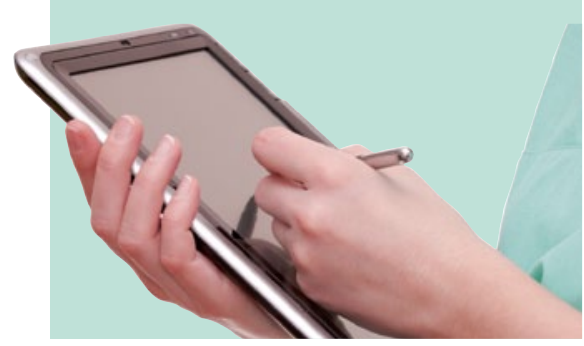

Knopfdruck reicht, um den ganzen Plan des Mitarbeiters neu zu berechnen.

Häufig wünscht das Klinikmanagement eine komplett automatische Dienstplanung auf Knopfdruck. Solche Programme gibt es und sie haben in Bereichen mit konstanten Dienstarten ihre Berechtigung. In der Pflege führt die automatische Dienstplanung jedoch häufig zu Unmut, da die menschliche, mitarbeiterorientierte Planung entfällt. Ein erfahrener Dienstplaner berücksichtigt für eine optimale Monatsplanung eben mehr als Regeln und Gesetze.

\section{Dienstplan-App für Smartphones?}

Unterschiedlichste Abstufungen in den Qualifikationen der Mitarbeiter machen zunehmend Planungen nach Bereichen oder Qualifikationen notwendig. So werden künftig für einzelne Planungsbereiche Mindestanforderungen definiert, die nur mit entsprechend qualifizierten Mitarbeitern besetzt werden können. Vielleicht profitieren die Mitarbeiter aber bald auch privat von Dienstplanprogrammen, beispielsweise wenn ein Dienstplanportal auf dem Smartphone hilft, Privattermine leichter zu planen oder Dienste unkompliziert zu tauschen.

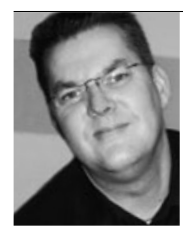

Heiko Mania, M.Sc., MBA Nordstr. 53, 50170 Kerpen mania@online.de www.gesundheits-it.com Der Autor ist ausgebildeter Krankenpfleger, Gesundheitsinformatiker und IT-Projekt-

manager. Seit vielen Jahren ist er als Dozent und IT-Trainer tätig. 